\title{
ШИРОКОПОЛОСНАЯ ПОЛОСКОВАЯ ПАТЧ-АНТЕННА В ВИДЕ МОДИФИЦИРОВАННОГО ПРЯМОУГОЛЬНИКА С U-ЩЕЛЬЮ В ЭКРАНЕ
}

\author{
ИРАМ НАДИМ, ДОНГ-Ю ЧОЙ
}

\author{
Университет Чосон, \\ Гванджу, Южная Корея
}

\begin{abstract}
Аннотация. Представлена широкополосная патч-антенна в виде модифицированного прямоугольного патча, который содержит три симметричных и асимметричный вырезы. В плоскость заземления вставлена $\mathrm{U}$-образная щель, которая способствует широкой относительной рабочей полосе частот $143 \%$ в диапазоне 2,9-17,5 ГГц. Модифицированный прямоугольный патч обеспечивает приемлемую всенаправленную диаграмму направленности на рабочих частотах с эффективностью излучения от 83 до 95\%. Максимально достижимое усиление составляет 5,48 дБ в указанном диапазоне частот. Электродинамическое моделирование осуществлялось с использованием высокочастотного симулятора структур HFSS, а схемное моделирование - с помощью программного обеспечения Advance Designing System (ADS). Эффект корпуса изучен при вертикальной ориентации антенны для оценки ее характеристик в присутствии проводящих тел. Предложенная архитектура антенны имеет небольшие электрические размеры $0,17 \times 0,11 \lambda$. Простая конфигурация и широкая полоса пропускания делают предлагаемую патч-антенну подходящим кандидатом для универсальных задач связи.
\end{abstract}

Ключевые слова: коэффициент перекрытия; метод видоизмененного экрана; коэффициент ширины полосы; сверхширокополосность; эффективная ширина полосы пропускания; BWR; DGS; BDR; UWB; EBIV

\section{1. ВВЕДЕНИЕ}

Потребность в широкополосных современных системах беспроводной связи растет из-за возрастающей пропускной способности и больших скоростей передачи данных. Современные значительные успехи в области беспроводной связи стимулировали спрос на малогабаритные недорогие антенны, которые могут работать на нескольких частотах с достаточно большой полосой пропускания.

Сверхширокополосность (СШП) - это важное свойство, позволяющее работать не- скольким задачам одновременно, таким как отслеживание местоположения, передача данных и георадар. В 2002 году Федеральная комиссия США по связи (FCC) объявила относительную полосу частот $110 \%$ (центральная частота 7,5 ГГц) и диапазон 3,1-10,6 ГГц для систем СШП радиосвязи. Преимуществом СШП систем является их невосприимчивость к эффектам многолучевого затухания, большая пропускная способность канала и высокая скорость передачи данных $[1,2]$.

\footnotetext{
* Это исследование профинансировано Программой фундаментальных исследований науки через Национальный исследовательский фонд Кореи (NRF), финансируемый Министерством образования (2016R1D1A1B03931806).
}

DOI: $10.20535 / S 0021347018120038$

() Ирам Надим, Донг-Ю Чой, 2018 


\section{REFERENCES}

1. HU, J.; YANG, J. "The adaptabilities of different UWB technologies to the FCC UWB emission limit," Proc. of Int. Conf. on Communication Software and Networks, ICCSN, Macau, China. IEEE, 2009, p.358-361. DOI: 10.1109/iccsn.2009.151.

2. FCC Online Table of Frequency Allocations. Accessed Feb. 2017. URI: https://transition.fcc.gov/oet/spectrum/table/ fcctable.pdf.

3. TANG, Hongyan; KE, Wang; WU, Runmiao; YU, Chao; ZHANG, Jian; WANG, Xiaotong. "A novel broadband circularly polarized monopole antenna based on C-shaped radiator," IEEE Antennas Wireless Propag. Lett., v.16, p.964, 2017. DOI: 10.1109/LAWP.2016.2615159.

4. DING, Kang; GAO, Cheng; YU, Tongbin; QU, Dexin "Broadband C-shaped circularly polarized monopole antenna," IEEE Trans. Antennas Propag., v.63, n.2, p.785, 2015. DOI: 10.1109/TAP.2014.2380437.

5. SAMSUZZAMAN, M.; ISLAM, Mohammad Tariqul. "A semicircular shaped super wideband patch antenna with high bandwidth dimension ratio," Microwave Opt. Technol. Lett., v. 57, n.2, p. 445,2015 . DOI: $10.1002 / \mathrm{mop} .28872$

6. MAHMUD, M.Z.; ISLAM, M.T.; SAMSUZZAMAN, M. "A high performance UWB antenna design for microwave imaging system," Microwave Opt. Technol. Lett., v.58, n.8, p.1824, 2016. DOI: $10.1002 / \mathrm{mop} .29924$.

7. CHOE, Hyeonhyeong; LIM, Sungjoon. "Ultrawideband compact U-shaped antenna with inserted narrow strip and inverted T-shaped slot," Microwave Opt. Technol. Lett., v.56, n.10, p.2265, 2014. DOI: $10.1002 / \mathrm{mop} .28566$.

8. TRIPATHI, Shrivishal; MOHAN, A.; YADAV, Sandeep. "A multinotched octagonal shaped fractal UWB antenna," Microwave Opt. Technol. Lett., v.56, n.11, p.2469, 2014. DOI: 10.1002/mop. 28629 .

9. SHAKIB, M.N.; MOGHAVVEMI, M.; MAHADI, W.N.L. "A low-profile patch antenna for ultrawideband application," IEEE Antemnas Wireless Propag. Lett., v.14, p.1790, 2015. DOI: 10.1109/LAWP.2015.2423931.

10. NEEBA, George; LETHAKUMARY, B. "A compact microstrip antenna for UWB applications," Microwave Opt. Technol. Lett., v.57, n.3, p.621, 2015. DOI: 10.1002/mop.28910.

11. TAHIR, Farooq A.; NAQVI, Aqeel H. "A compact hut-shaped printed antenna for super-wideband applications,"
Microwave Opt. Technol. Lett., v.57, n.11, p.2645, 2015. DOI: $10.1002 /$ mop. 29413.

12. GONG, Bing; REN, Xue Shi; ZENG, Ying Yin; SU, Lin Hua; ZHENG, Qiu Rong. "Compact slot antenna for ultra-wide band applications," IET Microwaves, Antennas \& Propag., v. 8, n.3, p.200, 2014. DOI: $10.1049 /$ iet-map.2013.0067.

13. SHAKIB, Mohammed Nazmus; MOGHAVVEMI, Mahmoud; MAHADI, Wan Nor Liza. "Optimization of planar monopole wideband antenna for wireless communication system," Plos one, v.11, n.12, 2016. DOI: 10.1371/journal.pone. 0168013

14. OJAROUDI, Nasser; OJAROUDI, Mohammad; GHADIMI, Noradin. "UWB omnidirectional square monopole antenna for use in circular cylindrical microwave imaging systems," IEEE Antennas Wireless Propag. Lett., v.11, p.1350, 2012. DOI: $10.1109 /$ LAWP.2012.2227137

15. OJAROUDI, Nasser. "Compact UWB monopole antenna with enhanced bandwidth using rotated L-shaped slots and parasitic structures," Microwave Opt. Technol. Lett., v.56, n. 1, p.175, 2014. DOI: $10.1002 / \mathrm{mop} .28055$.

16. SINGH, R.K.; PUJARA, Dhaval A. "A novel design of ultra-wideband quarter circular microstrip monopole antenna," Microwave Opt. Technol. Lett., v.59, n.2, p.225, 2017. DOI: $10.1002 / \mathrm{mop} .30271$.

17. KANJ, Houssam; POPOVIC, Milica. "Miniaturized microstrip-fed 'Dark Eyes' antenna for near-field microwave sensing," IEEE Antennas Wireless Propag. Lett., v.4, p.397, 2005. DOI: 10.1109/LAWP.2005.859377.

18. LIU, Jian. "Planar monopole notched ultra-wideband antenna with U-slot on metallic ground," Proc. of Int. Conf. on Applications of Electromagnetism and Student Innovation Competition Awards, 11-13 Aug. 2010, Taipei, Taiwan. IEEE, 2010, p.244-248. DOI: 10.1109/AEM2C.2010.5578792.

19. BORAH, J.; SHEIKH, T.A.; ROY, S. "Compact CPW-fed tri-band antenna with a defected ground structure for GSM, WLAN and WiMAX applications," Radioelectron. Commun. Syst., v.59, n.7, p.319, 2016. DOI: $\underline{10.3103 / \mathrm{S} 073}$ 5272716070050 .

20. KUMAR, A.; SINGH, M.K. "Band-notched planar UWB microstrip antenna with T-shaped slot," Radioelectron. Commun. Syst., v.61, n.8, p.371, 2018. DOI: $10.3103 / \mathrm{S} 07352$ 72718080058 . 